\title{
Volatile Organic Compound Discrimination using Nanostructured Polythiophene Sensors
}

\author{
Bo Li, S. Santhanam, L. Schultz, M. Jeffries-EL, \\ M. C. Iovu, G. Sauvé, J. Cooper, R. Zhang, J. C. \\ Revelli, A. G. Kusne, T. Kowalewski, L. E. Weiss, \\ R. D. McCullough, G. K. Fedder and D. N. Lambeth \\ Carnegie Mellon University, Pittsburgh, PA 15213, USA \\ $\underline{\text { lambeth@ece.cmu.edu }}$
}

\begin{abstract}
New synthesis methods have allowed us to make many semiconducting polythiophenes polymers with different side and end groups. Also, co-polymers combining a polythiophene chain attached to another polymer chain were synthesized. This design freedom brings a new dimensionality to the sensing properties of the materials. Single chip micro sensor resistor arrays, utilizing multiple polymers, were fabricated and then tested in an automated system. The sensors demonstrated ppm level sensitivity to various volatile organic compounds (VOCs) including both polar and non-polar materials. Polymers with different chemical structures show strong selectivity to different VOCs. By applying pattern recognition algorithms, the sensor response clearly discriminates between the tested VOCs allowing us to conjecture as to the role molecular modification have in determining response to specific VOCs.
\end{abstract}

\section{INTRODUCTION}

A low-cost, low-power, and portable device for volatile organic compound (VOC) detection and identification is needed for a number of applications [1]. One of the most difficult challenges is to find materials that have good sensitivity and strong analyte selectivity. While there has been limited success in sensor development for greenhouse gases $\left(\mathrm{CO}_{2}, \mathrm{CH}_{4}, \mathrm{~N}_{2} \mathrm{O}\right.$, NO and $\left.\mathrm{CO}\right)$, the technology for detection of VOCs remains weak due to the similarity of chemical composition and structure by small molecules. Existing VOC sensing materials include semiconducting metal oxides, carbon black-polymer composites, and conjugated polymers ( $\mathrm{CPs}$ ), each having its own advantages and drawbacks. Due to their potential for mass production, room temperature operation and compatibility with microfabrication techniques, the use of CPs is promising. However, low sensitivity and selectivity to non-polar analytes has been a major drawback in the past.

With large possible number of chemical variants the polythiophenes are some of the most promising CPs for sensor arrays [2]. Easy molecular structure modification endows them with extended chemical selectivity. Their solution solubility enables the use of ink-like printing for batch device fabrication. Our recent advances in synthesis methods [3]-[5] have allowed us to make many semiconducting polythiophene polymer variants with various side groups (R-groups), end groups and copolymers, which

\author{
J. L. Snyder \\ Pittsburgh Research Laboratory \\ National Institute of Occupational Safety and Health* \\ Pittsburgh, PA 15236, USA
}

have a secondary polymer chain attached to the end, or inserted into the body, of the primary polythiophene chain. These molecular structure modifications enabled interesting chemical sensitivity and selectivity. In this work, with only 10 of the 30 polythiophene polymers synthesized to date, we endeavor to illustrate the role of the polymer and analyte's chemical structure to sensing responses.

\section{EXPERIMENT}

\section{A. Material Selection}

Table I shows the representative molecular structures selected for this study. P3HT, PDDT, and PMEEM are polythiophene polymers with different side chains. P3HT, benzyl terminated P3HT, and bromoester terminated P3HT are polythiophene polymers with different end groups. PHTb-PBA, PHT-b-PMA, and PHT-b-PS are polythiophene based block copolymers with different secondary polymer chains. PDDT-ran-PMT and PHT-ran-PEOHT are random copolymers with different secondary chains randomly inserted into the primary polythiophene chain. The polymers were dissolved in trichlorobenzene at $5 \mathrm{mg} / \mathrm{ml}$ and filtered with a $0.4 \mu \mathrm{m}$ PTEF syringe filter prior to sensor fabrication.

\section{B. Sensor Fabrication}

Chemresistor devices were prepared on conductive $\mathrm{N}+$ silicon substrates with a thermally grown $700 \mathrm{~nm}$ insulating $\mathrm{SiO}_{2}$ surface. A $5 \mathrm{~nm}$ layer of Ti covered by a $50 \mathrm{~nm}$ layer of $\mathrm{Au}$ was sputter deposited and patterned as the electrodes. A computer-controlled ink-jet deposition system was used to deposit the polymer solutions onto $200 \mu \mathrm{m}$ diameter spiral electrode pairs with conductor widths and spacing of $3 \mu \mathrm{m}$. This circular geometry best matched the circular spots produced by the inkjet. After limited testing, $10-60$ picoliter drops were found to optimize the chemresistor sensor response. The sensors were vacuum annealed at $100{ }^{\circ} \mathrm{C}$ for 1 hour, then place in the test chamber and conditioned by flowing pure $\mathrm{N} 2$ over them for 12 hours before vapor testing at room temperature.

\section{Sensor Testing}

Ten VOC vapors; methanol, iso-propanol, ethanol, acetone, hexane, cyclohexane, methylenchloride, 
acetonitrile, toluene and benzene were studied. Depending upon the polymer, and for each vapor, either 5 or 6 concentrations were introduced into the sensor test chamber via a $1 \frac{1}{1} \mathrm{~min}$ pure nitrogen carrier gas. Labview based computer automated flow controllers delivered the vapors at an exposure schedule of 10 minutes on and 10 minutes off while a Keithley 2700 multimeter simultaneously recorded the currents flowing through 6 individual chemresistor sensors at 5 second sampling intervals.

TABLE I

MOLECULAR STRUCTURE OF TESTED POLYMERS

\begin{tabular}{cc}
\hline P3HT \\
\hline Poly(3-hexylthiophene)
\end{tabular}

\section{RESULTS}

\section{A. Polymer Nanostructure}

Atomic Force Microscopy (AFM), Fig. 1, was used to investigate the morphology of our polymers cast from a slowly evaporating solvent onto an oxidized silicon substrate. Other reports $[6,7]$ indicate that due to the $\pi-\pi$ interchain stacking of the polythiophene molecules dense, crystalline nanowire structures form from the P3HT homopolymer and that the electronic properties are correlated to the molecular weight and microstructure. However, only the polythiophene portion of some of the copolymer samples stacks as a nanowire structure, while the secondary polymer, of the copolymer, remains amorphous. This results in an apparent unordered region between the nanowires. Likewise, it is believed that different side and end groups result in different lattice spacing down the length of the nanowires, as well as, a varied polycrystalline structure.

Film morphology has an important role in determining the material's sensing properties [8]. This is especially true when comparing the crystalline structures to those of a totally amorphous structure. These variations, such as, lattice parameters, porosity, nanowire length and width, and grain boundary thickness, alter the electrical conduction mechanisms and so alter the sensing sensitivity, selectivity, and respond time based upon the analyte. It also appears that this crystalline nanostructure improves the sensitivity of CPs to non-polar analytes [9].

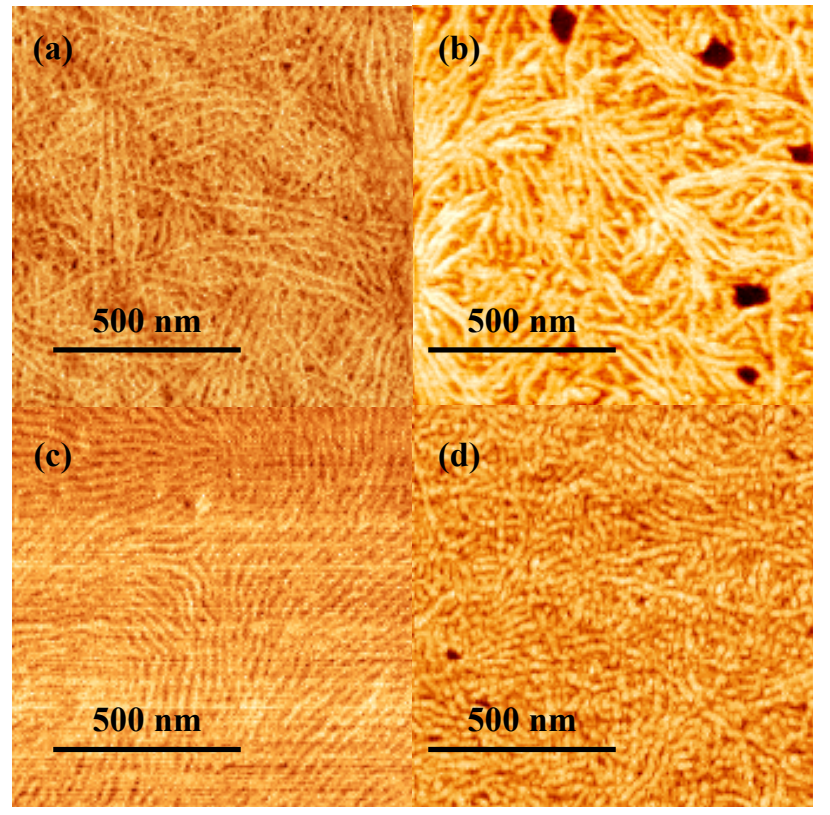

Fig. 1. Tapping mode AFM phase images illustrate the various nanostructures of (a) P3HT, (b) PHT-b-PS, (c) PHT-b-PBA, (d) PHT-bPMA thin films.

\section{B. Sensor Performance}

Fig. 2 shows the normalized temporal conductance response of different polymers to methanol and benzene. There are several interesting observations: 1) almost all of the polymers demonstrated a fast chemical response time, typically $t_{90}<30$ second at around 200 ppm analyte concentration; 2) the sensors recovered completely to their baseline after vapor exposure; 3) for low concentrations, the responses were nearly linear with analyte concentration; 4) different polymers showed different sensitivity amplitude; 5) interestingly, the responses of different polymers can have different signs, with some polymers showing positive conductance change and some showing negative change.

\section{Principal Component Analysis}

Principal component analysis (PCA) was used to illustrate sensor array response patterns. Different polymer sensor responses, to vapor concentrations of $200 \mathrm{ppm}$, were used as the PCA input to XLStat 7.5 statistical software. For 
example, the score plot of the first two principal components (Fs) is shown in Fig. 3. The sensor array has discriminated these VOCs via separation of each data point. There is also a clear correlation between the PCA output and physical properties of the VOCs. F1 is correlated to the dipole moment of the VOCs by yielding a negative F1 value to the non-polar VOCs and a positive value to polar ones. F2 is a positive value for non-polar VOCs with a benzene ring in their structures and negative value for others.
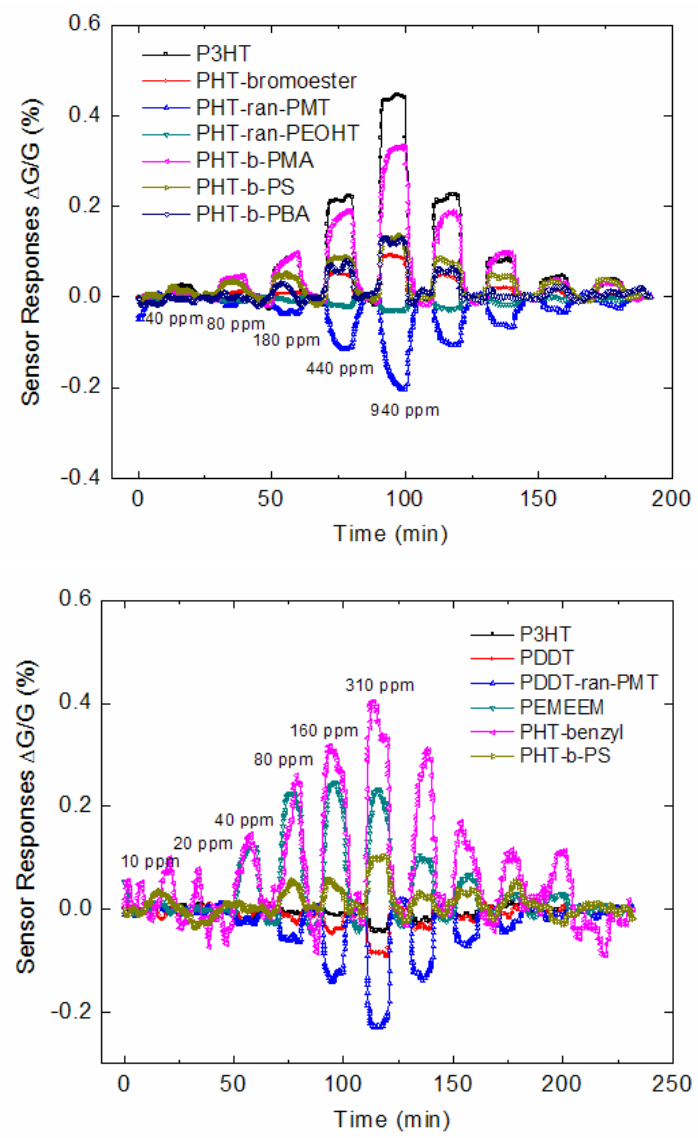

Fig. 2. Sensor responses of tested polymers to VOC vapors plotted versus time: (a) methanol; (b) benzene.

\section{DISCUSSION}

Wonderfully, the response of the different polymers to the VOCs is different. This means the film microstructure and the molecular structure of the polythiophene polymers, which is due to the different side and end groups, have a strong influence on their sensing properties. Generally, the sensing behaviors are related to some molecular interactions such as bond type and angle, dipole interaction and the van der Waals force between the analyte and polymer. But, for crystalline films it is also due to the microstructure. These interactions possibly modify the charge transportation inside the polymer molecule, or inside or between the nanowires. At the room temperature regime, both inter and intrananowire carrier hopping is believed to be the conduction mechanism for CPs. Parameters like barrier height and distance between delocalized states determine the current density [10]. In the following, we propose some possible sensing mechanisms for the polythiophene polymers due to the different molecular structures.

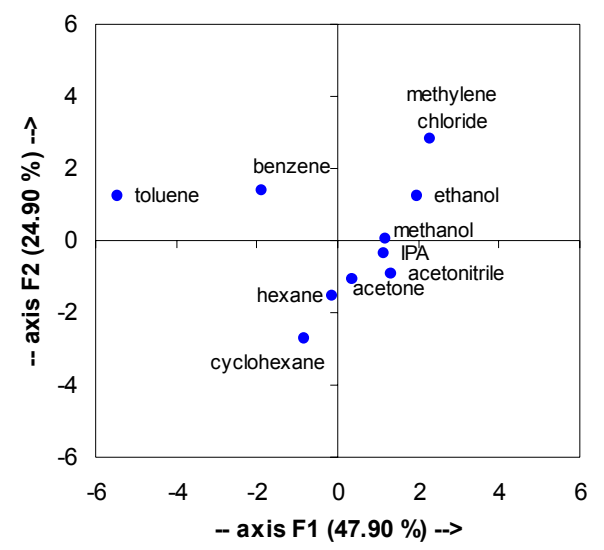

Fig. 3. Principal component score plot of responses of 10 polymers to 10 VOC vapors at $200 \mathrm{ppm}$.

\section{A. Side Group Effects}

The three polymers, P3HT, PDDT and PMEEM, have different side groups. Their resposne patterns are shown in Fig. 4(a). P3HT and PDDT both have an alkyl side group. And they show similar reponse patterns, a positive response to polar analytes and a negative response to non-polar analytes. However, PMEEM showed positive responses to all VOCs. Probably the molecular interactions between the dipolar alkyl side group and polar analytes cause compaction of the nanowires, thus reducing the hopping distance and so increasing the polymer conductance. While non-polar analytes, such as toluene and benzene, which are actually solvents for these polymers, disolve into the polymer and separate both the polymer molecules and crystalline structures. Hence, the carrier hopping distance increases and conductivity decreases. PMEEM, which has a side group containing oxygen, has a much higher dipole moment than the alkyl side group polymers. A stronger dipole-dipole interaction occurs when exposed to all analytes. This is probably the reason that this polymer generally shows possitive response to VOCs.

\section{B. End Group Effects}

The three polymers, P3HT, PHT-benzyl, and PHTbromoester have different end groups. Their response patterns are shown in Fig. 4(b). While the PHT-bromoester showed a similar response to P3HT, PHT-benzyl showed a significantly different response. PHT-benzyl had a huge positive response to toluene and benzene. Here, the nonpolar properties of toluene and benzene result in a weak dipole-dipole interaction. Since the benzyl end group has a benzene ring, it is plausible that the van der Waals force is the main interaction between analytes and end groups with a 
similar structure to the analytes. This interaction is attractive, hence, it brings the polymer molecules closer and reduces the delocalized state distance, increasing the conductivity.
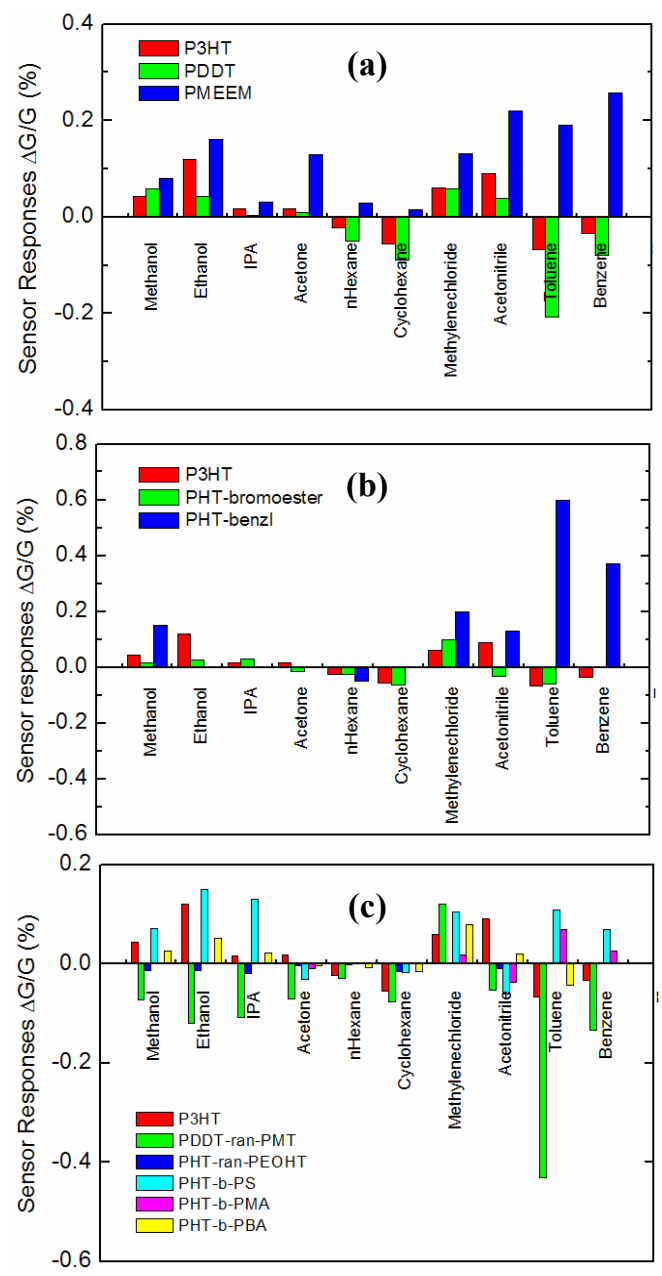

Fig. 4. Molecular structure effects on response pattern: (a) side chain effects, (b) end group effects, (c) copolymer effects.

\section{Copolymers}

Since the copolymers have a secondary polymer chain inside or at the end of the polythiophene polymer, the sensing properties will greatly depend on the structure of the secondary polymer chain. For example, PHT-b-PS shows a strong positive response to toluene and benzene, which are solvents for, and have a similar chemical structure to the secondary polymer, polystyrene. Again, the van der Waals force tends to bring the similar molecules closer together reducing the charge hopping distance. As for PDDT-ranPMT and PHT-ran-PEOHT, the secondary chain was inserted inside the primary polymer in random segment lengths. They showed negative response for most VOCs. We tend to believe that the interaction of the analyte molecules and alternating side groups can twist the copolymer molecules. Thus, the electronic coupling between adjacent monomer units is interfered with and the current density along molecule chain decreases [11].

\section{CONCLUSION}

In summary, we have demonstrated the richness of using the family of polythiophene based conductive polymers and copolymers with various side and end groups as VOC vapor sensors. The sensing results showed a variety of new response patterns, which can potentially greatly enhance the discrimination between VOCs. A few of the roles of the chemical interactions between the vapors and the variety of molecular chemistries of the polythiophenes have been conjectured as to the cause of the electrical property changes. This effort provides encouragement that with further work along these lines one will be able to predict which polymer chemical structure to utilize for optimum response to a given analyte.

\section{REFERENCES}

[1] A. K. Srivastava, "Detection of volatile organic compounds using $\mathrm{SnO} 2$ gas sensor array and artificial neural network," Sens. Actuators $B$, vol. 96, pp. 24-37, 2003.

[2] L. Torsi, A. Tafuri, N. Cioffi, M. C. Gallazzi, A. Sassella, L. Sabbatuni, P. G. Zambonin, "Regioregular polythiophene field-effect transistors employed as chemical sensors," Sens. Actuators B, vol. 93, pp. $257-262,2003$.

[3] Malika Jeffries-EL, Genevieve Sauvé, Richard D. McCullough, "InSitu end-group functionalization of regioregular poly(3alkylthiophene) using the Grignard metathesis polymerization method," Adv. Mater., vol. 16, pp. 1017-1019, 2004.

[4] Lei Zhai, Richard L. Pilston, Karen L. Zaiger, Kristoffer K. Stokes, Richard, D. McCullough, "A Simple method to generate side-chain derivatives of regioregular polythiophene via the GRIM metathesis and post-polymerization functionalization," Macromolecules, vol. 36, pp. 61-64, 2003.

[5] Mihaela C. Iovu, Malika Jeffries-EL, Elena E. Sheina, Jessica R. Cooper, Richard D. McCullough, "Regioregular poly(3alkylthiophene) conducting block copolymers," Polymer, 2005, in print.

[6] Zhenan Bao, Ananth Dodabalapur, and Andrew J. Lovinger, “ Soluble and processable regioregular poly(3-hexylthiophene) for thin film field-effect transistor applications with high mobility," Appl. Phys. Lett., vol. 69, pp. 4108-4110, 1996.

[7] Rui Zhang, Bo Li, Jessica Copper, et al., "Nanostructure Dependence of Field-Effect Mobility in Regioregular Poly(3-hexylthiophene) Thin Film Field Effect Transistors," J. Am. Chem. Soc., in print.

[8] L. Torsi, A. J. Lovinger, B. Crone, T. Someya, A. Dodabalapur, H. E. Katz, and A. Gelperin, "Correlation between oligothiophene thin film transistor morphology and vapor responses," J. Phys. Chem. B, vol. 106, pp. 12563-12568, 2002.

[9] Bo Li, Rui Zhang, Suresh Santhanam, et al., "Chemical sensing of highly ordered polythiophene nanowire structures," unpublished.

[10] Erik Johansson, Sven Larsson, "Electronic structure and mechanism for conductivity in thiophene oligomers and regioregular polymer," Synth. Met., vol. 144, pp. 183-191, 2004.

[11] Ferdinand C. Grozema, Piet Th. Van Duijnen, et al., "Intramolecular charge transport along isolated chains of conjugated polymers: effect of torsional disorder and polymerization defects," J. Phys. Chem. B, vol. 106, pp. 7791-7795, 2002.

* The findings and conclusions in this report are those of the authors and do not necessarily represent the views of the National Institute for Occupational Safety and Health. 